\title{
Bidirectional Interference between Speech and Postural Stability in Individuals with Parkinson's Disease
}

\author{
Christopher Dromey \\ Brigham Young University, dromey@byu.edu \\ Eon Jarvis \\ University of Utah \\ Stuart Sondrup \\ University of Utah \\ Shawn Nissen \\ Brigham Young University \\ K. Bo Foreman \\ Ebollirowsthissofruetalditional works at: https://scholarsarchive.byu.edu/facpub \\ Part of the Communication Sciences and Disorders Commons
}

See next page for additional authors

Original Publication Citation

Dromey, C., Jarvis, E., Sondrup, S., Nissen, S., Foreman, K.B. \& Dibble, L.E. (2010). Bidirectional interference between speech and postural stability in individuals with Parkinson's disease. International Journal of Speech-Language Pathology, 12, 446454.

\section{BYU ScholarsArchive Citation}

Dromey, Christopher; Jarvis, Eon; Sondrup, Stuart; Nissen, Shawn; Foreman, K. Bo; and Dibble, Leland E., "Bidirectional Interference between Speech and Postural Stability in Individuals with Parkinson's Disease" (2010). Faculty Publications. 1781.

https://scholarsarchive.byu.edu/facpub/1781

This Peer-Reviewed Article is brought to you for free and open access by BYU ScholarsArchive. It has been accepted for inclusion in Faculty Publications by an authorized administrator of BYU ScholarsArchive. For more information, please contact ellen_amatangelo@byu.edu. 


\section{Authors}

Christopher Dromey, Eon Jarvis, Stuart Sondrup, Shawn Nissen, K. Bo Foreman, and Leland E. Dibble 
Bidirectional interference between speech and postural stability in individuals with Parkinson's disease

Christopher Dromey ${ }^{1}$

Eon Jarvis ${ }^{2}$

Stuart Sondrup ${ }^{2}$

Shawn Nissen ${ }^{1}$

K. Bo Foreman ${ }^{2}$

Leland E. Dibble²

${ }^{1}$ Brigham Young University

Department of Communication Disorders

${ }^{2}$ University of Utah

Department of Physical Therapy

Contact Author:

Christopher Dromey, PhD

Department of Communication Disorders

133 John Taylor Building

Brigham Young University

Provo, UT 84602 USA

tel: 801-422-6461

fax: 801-422-0197

email: dromey@byu.edu 


\begin{abstract}
Because people frequently talk while engaged in other activities, and because Parkinson's disease (PD) is known to diminish multi-tasking performance, we examined dual task interference between speaking and postural stability in 9 individuals with PD, 7 agematched and 10 healthy young controls. Participants repeated a target utterance and performed a rise to toes task in both single and dual task conditions. Diphthong transitions were measured from audio recordings and postural variables reflecting planning, coordination, and stability were derived from a multi-camera motion capture system and force plate recordings. Thus, sensitive measures of both speech and postural control were obtained. The group with PD performed more poorly than both control groups for the isolated postural task, but their single task speech measures did not differ from the controls, in spite of listener ratings which indicated mild to moderate dysarthria severity. The group with PD showed evidence of bidirectional dual task interference in that there were reduced diphthong extents and slopes along with smaller, slower, and less stable postural movements. These results indicate that concurrent performance of speech and a challenging postural control task impairs speech and postural stability in persons with PD and may result in greater risk during daily activities.
\end{abstract}




\section{Introduction}

Hypokinetic dysarthria associated with Parkinson's disease (PD) is characterized by monopitch and monoloud speech, hypophonia, imprecise consonants, speech rhythm disturbances, and rate variability (Adams, 1996; Gentil \& Pollak, 1995). Pharmacological treatments for PD, which have led to improved limb and axial function, have shown mixed results for speech. While some authors have reported improvements with levodopa (Nakano, Zubick, \& Tyler, 1973), others have found either minimal or no effect from medication on a variety of perceptual speech features (Ho, Bradshaw, \& lansek, 2008). Thus, behavioral intervention is currently the most promising means of improving speech in individuals with PD. Because the success of such treatments depends on effort from the person with dysarthria, clinicians need to be aware of potential difficulties with attention and executive function, which have been found to be impaired in individuals with PD (Wu \& Hallett, 2008).

It has been suggested that people with PD perform motor tasks with reduced automaticity (Galletly \& Brauer, 2005; Yogev, Giladi, Peretz, Springer, Simon et al., 2005). They may rely on frontal cortical mechanisms to compensate for their basal ganglia deficits by more deliberately focusing attention on activities that in the healthy individual are completed with little effort (Morris, lansek, Smithson, \& Huxham, 2000; Rochester, Nieuwboer, Baker, Hetherington, Willems et al., 2008). The suggested cortical compensatory mechanisms described in the gait literature (Camicioli, Oken, Sexton, Kaye, \& Nutt, 1998; Rochester et al., 2008) may also be responsible for speech improvements in the Lee Silverman Voice Treatment, where patients concentrate on the single target of loudness (Ramig, Fox, \& Sapir, 2004). However, the accompanying deficits in executive function can add to the challenge of 
maintaining outside the clinic the speech production gains achieved during therapy. Activities of daily living often require a considerable amount of an individual's attention, thus their speech could be compromised when performed at the same time. Because people often speak while doing other tasks, dual task experiments can be important to help us understand the impact of the disease in everyday situations.

The dual task literature suggests that decrements in performance often occur in one or both activities as limited attentional resources are divided across tasks. This phenomenon is referred to as task interference. Brown and Marsden (1991) suggested two reasons why there may be greater dual task interference in people with PD than in healthy individuals. First, those with PD may have fewer attentional processing resources available because of their neurological illness. Tasks that rely on normal basal ganglia function now require more conscious attention to complete, with reliance on cortical mechanisms (Camicioli et al., 1998). Secondly, research in limb motor control suggests that it is difficult for individuals with PD to switch between tasks (Weiss, Stelmach, \& Hefter, 1997). A recent neuroimaging study revealed that when individuals performed two tasks simultaneously, those with PD had increased brain activation compared to neurologically healthy individuals, even after a period of training with both tasks (Wu \& Hallett, 2008).

In contrast to the number of studies examining gait during dual-tasking in people with PD (Galletly \& Brauer, 2005; Rochester et al., 2008; Wu \& Hallett, 2008), few researchers have studied interference effects during tasks that are more specific to postural stability. Marchese and colleagues (Marchese, Bove, \& Abbruzzese, 2003) compared the impact of a cognitive secondary task (subtraction by $3 \mathrm{~s}$ ) to a motor secondary task (thumb opposition to the 2nd-5th 
digit in sequence) on postural stability during standing. They found decreased postural stability with the addition of either secondary task during quiet standing compared to quiet standing alone. This indicated to the authors that postural stability deficits were present in individuals with PD during dual task conditions independent of the type of secondary task, although the performance of the secondary task was not measured in this study. A more recent study reported reduced postural stability when individuals with PD produced a monologue while standing on a force plate. In this experiment speaking served as a secondary task and no speech variables were quantified (Holmes, Jenkins, Johnson, Adams, \& Spaulding, 2010).

When examining postural stabilization as one of the dual tasks, there is disagreement in the literature regarding how individuals with PD prioritize task performance. In one study (Morris et al., 2000) the authors suggested that the individuals with PD placed more attention on maintaining stability than on reciting words, thus prioritizing stability, whereas the healthy age-matched group did not. However, Bloem and colleagues (Bloem, Grimbergen, van Dijk, \& Munneke, 2006) demonstrated bidirectional interference in healthy young adults, participants with PD, and age-matched controls. Whereas the healthy young and age-matched controls made more cognitive errors during dual task performance, the group with PD made more motor errors. This suggested to the authors that the healthy young and age-matched participants chose a posture first strategy, which allowed some loss of performance on the nonpostural task in order to maintain balance. On the other hand, the group with PD chose a less safe strategy by not prioritizing the maintenance of postural stability.

The existing literature shows that most dual task studies of PD have made more detailed measures of a primary task, with less sensitivity in the measurement of the secondary or 
competing task, making it difficult to clearly examine bidirectional interference effects. The present study set out to provide detailed measures of both speech and postural performance in order to quantify the extent of bidirectional interference in people with PD relative to agematched and younger control participants. On the basis of a model of limited attentional resources for motor tasks in PD (Cutson, 1994), we hypothesized that 1) those with PD would demonstrate speech and postural performance deficits relative to control participants; 2) in each group, single task performance of speech and postural tasks would exceed that in dual task conditions; and 3) the bidirectional interference effects of speech and posture in people with PD would exceed those found in control participants.

\section{Methods}

\section{Participants}

A sample of 9 individuals with mild to moderate PD, 7 age-matched controls, and 10 healthy young controls consented to participate. The ages of the control groups as well as disease specific characteristics of the PD group are summarized in table 1 . Individuals with PD presenting for traditional outpatient physical therapy or a community based risk reduction program were recruited for this study. Inclusion criteria included a confirmed medical diagnosis of idiopathic PD, and the physical and cognitive abilities to actively participate in the study procedures. Exclusion criteria included individuals who were cognitively unable to understand or follow study instructions, individuals with significant orthopedic (i.e. fracture, moderate to severe osteoarthritis) or neurological (i.e. stroke, traumatic brain injury, neuropathy) injury. Neurologically healthy individuals who were age- and gender-matched to the PD group were 
recruited from the community as a control group. In addition, ten university students were recruited for a healthy young control group. The study was approved by the Institutional Review Board at the University of Utah and all participants gave their signed consent.

\section{Instrumentation}

All testing was conducted at the Motion Capture Core Facility at the University of Utah Department of Physical Therapy. During the speaking tasks each participant wore a headset microphone (Logitech, Inc.) while the audio signal was recorded into a software program (Audacity, version 1.3.5) on a lab computer at a sample rate of $44.1 \mathrm{kHz}$.

Prior to the collection of the balance and postural control data, static and dynamic calibrations of the motion analysis system (An 8 camera Vicon Kinematic Analysis System and an Advanced Medical Technologies triaxial force plate) were performed. For testing, participants wore black tight-fitting clothing and no shoes. Passive reflective markers were placed on bony prominences utilizing a standardized gait analysis marker set (Plug-In Gait marker set, Vicon Motion Systems). Participants were then asked to stand in a comfortable position on the force plate, which was covered with paper. Each individual's feet were traced with a marking pen and these tracings were used as the starting position for all trials. Data were collected at a sample rate of $200 \mathrm{~Hz}$ for the cameras and $1 \mathrm{kHz}$ for the force plate from the time the starting stimulus was given until after the movement task was completed. Participants were supervised closely during all trials in order to prevent falls.

\section{Procedure}

Each participant performed the tasks under both single and dual task conditions with the order of testing determined by random drawing. The speaking task involved the production 
of two target sentences, the boot on top is packed to keep and the boy gave a shout at the sight of the cake. These were selected because they included the corner vowels and several diphthongs, which allowed inferences about lingual excursions via measurement of the first and second formants. In order to minimize postural demand during the single task speaking condition, the participant spoke while sitting in a chair with back support. The sentences were read from a sheet of paper at a comfortable rate and loudness without an auditory start cue.

In order to globally characterize the postural control of participants, their fall history during the previous year was noted. In addition, individual trials were screened qualitatively to insure that participants could perform the task. The postural control task was a rise to toes task performed with a stationary base of support. This task was selected because it required participants to voluntarily move from a stable (full foot to ground contact) to an unstable posture (only forefoot to ground contact). Specifically, upon hearing an auditory start cue, the participants rose to their toes as quickly as possible and they then maintained a stable posture. Standardized instructions were given to the participants, "at the sound of the beep, rise to your toes as fast as you can and stay as high and stable as you can". Each trial lasted 5 seconds, and then the participant descended to a natural standing posture. In the single task condition, the participant performed the postural control task without speaking. For the dual task condition, the participant performed the tasks concurrently, remaining on their toes for 5 seconds or until the target utterances were completed. Five trials of each condition were gathered. Testing was performed one to two hours after each patient had taken his/her anti-PD medications so that pharmacological influences would be consistent throughout the testing. 


\section{Data Processing and Analysis}

The independent variables used for analysis were group assignment (PD, age-matched control, young control), and task condition (single task, dual task).

The audio recordings were saved as wav files. In order to characterize the speech of the PD group, their dysarthria severity was independently rated by 2 graduate students in speechlanguage pathology on a scale ranging from 1 (normal) to 10 (severely dysarthric). The recordings were analyzed with Praat software (version 5.0.47). First, the diphthongs were identified from the waveform and spectrographic displays, and the segmentation points were confirmed by audio playback. Formant tracks were visually inspected for accuracy and were exported as text files with listings at $1 \mathrm{~ms}$ intervals. These files were imported into custom Matlab routines that divided the diphthong duration into 8 equally-sized adjacent windows. The mean formant values within the second and seventh windows were operationally defined as the onglide and offglide frequencies. The difference between these onglide and offglide values was the formant transition extent for F1 or F2. The time difference between the windows was the diphthong transition duration, and the slope was calculated by dividing the transition extent by the transition duration for F1 and F2. For the purposes of the present study, data from the diphthongs /כI/ (boy) and /eI/ (cake) are reported. The formant transition durations, extents, and slopes were selected as dependent measures because they have been associated in previous studies with changes in articulatory function in speakers with PD (Dromey, Ramig, \& Johnson, 1995). It was reasoned that they would be sensitive to dual task interference if the concurrent postural task were to negatively impact articulatory performance by reducing lingual excursions. 
The kinematic and kinetic data were initially saved in Vicon proprietary format. The marker location trajectories and force data were exported as text files. Custom written Visual Basic macros in Microsoft Excel were then used to extract the variables of interest. Two specific biomechanical variables, center of mass (COM) and center of pressure (COP) were core to the data analysis. Center of mass is a point in the body about which the mass is evenly distributed, and was computed from the reflective markers on the body surface on the basis of anthropometric norms. For the purposes of the present study, the COM variable was defined as vertical projection of the COM onto a point on the floor directly below the computed center of mass for the body, and thus did not have a vertical component. The center of pressure was computed from the output of the force plate upon which the participant stood. It represents the weighted average of all the vertically oriented forces acting on the plate.

The dependent variables derived from the postural task were reaction time, COM-COP difference, COP velocity, heel height, and coefficient of variation of heel height. Reaction time was operationally defined as the latency between the audio start cue and the time when the elevation of the heel was two standard deviations above the rest position on the force plate. Based on previous research, reaction time was utilized as an overall measure of the preparatory processes inherent to the construct of motor planning (Cutson, 1994).

Two variables were used to reflect the construct of postural coordination. The derived variable COM-COP difference was used to reflect postural coordination in the sagittal plane prior to the onset of heel movement, while COP velocity was used to reflect postural coordination during the ascent phase of the rise to toes movement. The COM-COP variable was operationally defined as the maximal sagittal plane difference between the locations of the 
COM and the COP during the rise to toes task. This variable capitalizes on the biomechanical relationship between the COP and the COM variables in that COP must change (as a person adjusts the forces transmitted downward through the feet) in order to change their posture and thus their COM (Martin, Shinberg, Kuchibhatla, Ray, Carollo et al., 2002). The second postural coordination variable, the center of pressure (maximum) velocity, was derived from the steepest part of the COP slope during the rise to toes task, and thus represented the most rapid change in displacement of the COP. The construct of postural stability was characterized by two variables, average heel height or displacement and heel height coefficient of variation. Heel height was operationally defined as the average vertical displacement of the heel marker of the dominant leg during the portion of the task where the participant was attempting to maintain stability on their toes. Heel height coefficient of variation was operationally defined as the standard deviation of the heel vertical displacement divided by the average heel height during the on toes period. Figure 1a shows how the variables were defined on the recordings from the force plate and the motion tracking system. This recording was from a healthy young participant.

All statistical analyses were performed with SPSS 16 software (SPSS Inc). Between group comparisons on all dependent variables in the single task condition were performed using separate one-way ANOVAs with Tukey's HSD post hoc analyses calculated when appropriate. For each dependent variable, single and dual task differences were compared within the PD and control groups using separate repeated measures ANOVAs, and effect sizes were computed during this analysis (Cohen, 1992; Lipsey, 1990). The level of significance was set at $p<0.05$. 


\section{Results}

\section{Group Differences}

There were no significant differences between groups for the speech variables for /כI/.

The duration of /ei/ was shorter for the healthy young speakers than for the other two groups.

The F2 extent for /eI/ was smaller for the healthy young speakers than for those with PD (table 2).

On the postural measures, COP velocity was higher for the healthy young participants than for both of the older groups. The healthy young participants had a larger COM-COP difference than both of the older groups. Heel height was higher for the healthy young participants compared to both the PD and age-matched groups (table 3 ). Figure $1 \mathrm{~b}$ shows a postural recording from a single task trial performed by an individual with PD, revealing visible differences in performance compared with the healthy young participant in figure 1a.

\section{Single/Dual Task Differences}

For the diphthong /כI/ there was a decrease in F1 extent and F1 slope for the dual task condition only for the speakers with PD. For /ei/ the F2 extent and slope decreased significantly for the dual task condition in the speakers with PD, but not for the younger or older control speakers. Effect sizes were in the medium to large range (Cohen, 1992) for each of these dual versus single task differences (table 2 ).

For the postural variables, the reaction time increased for all three groups when performing the speech and postural tasks together, although these differences were only significant at $\mathrm{p}<.05$ for the healthy young and PD groups. Heel height decreased significantly for 
the PD group and the age-matched control participants in the dual task condition. For the healthy young control participants, the coefficient of variation of heel height increased for the dual task condition. The effect sizes were in the medium to large range (table 3). Figure 1c shows the postural record in the dual task condition for an individual with PD, revealing performance instability beyond that revealed in the single task condition in figure $1 \mathrm{~b}$.

\section{Discussion}

The purpose of this study was to quantify both speech articulation and postural motor performance independently and then with both tasks performed concurrently in order to examine potential prioritization and bidirectional interference of the tasks in individuals with PD compared to age-matched and younger healthy controls. Relative to our hypotheses, we found that, 1) participants with PD demonstrated postural motor performance deficits relative to neurologically healthy age-matched controls as well as healthy young controls. However, there were no statistical differences between participant groups on the speech variables; 2) Dual task situations generally resulted in the degradation of certain measures speech articulation and postural motor performance relative to single task performance; and 3) participants with PD exhibited greater bidirectional interference effects of speech articulation and postural motor performance than the two control groups.

\section{Performance comparison between groups}

When compared to healthy young and age-matched individuals, the PD group performed at lower levels on the postural measures. This would be expected, because reduced postural stability is one of the symptoms of the disease (Marchese et al., 2003). However, their 
performance on the speech measures was not substantially worse than for the controls, in spite of the speakers with PD being rated as mildly to moderately dysarthric by the listeners. The acoustic speech variables showed only one significant difference (diphthong duration) between the groups during the single task condition. People with PD have been reported to have speech rate impairments (either slower or faster), and the longer segment durations found in the present study are consistent with the findings of reduced rate, which is a common finding in many dysarthric speakers (Adams, Weismer, \& Kent, 1993).

The lack of significant differences in the diphthong extent and slope measures was surprising in light of previous research (Ackermann \& Ziegler, 1991) which has suggested reduced articulatory excursions in speakers with PD. The present findings may be an indication that the repetitious speech task in the present study may not have been challenging enough to elicit performance that would distinguish between the groups. Therefore, the selection of stimulus sentences with a different set of target phonemes might have revealed acoustic evidence of articulatory undershoot not found in the present study. Even though the speakers with PD were judged to be impaired by our listeners, these ratings may have been influenced more by phonatory parameters and less by the articulatory behaviors reflected by the formant measures used for analysis. Previous accounts have suggested that spectral features of the laryngeal sound source may account for perceptible differences between the speech of individuals with PD and controls (Dromey, 2003).

In contrast to the lack of speech differences, there were significant differences between the groups on the postural measures (see table 3). There was a trend of increasingly impaired performance for each variable stepwise from the healthy young to the age-matched controls to 
the PD group. The significant differences in COP velocity, COM-COP difference, and heel height show that the individuals with PD had smaller and slower movements than the controls. This is consistent with previous research reporting that people with PD do not perform balance or gait tasks as well as age-matched counterparts or healthy young adults (Bloem et al., 2006; Canning, 2005; Morris et al., 2000).

\section{Single task performance compared to dual task performance}

The PD group was affected more than the control groups by the dual task condition for both speaking and postural tasks, suggesting that they are more likely than the healthy individuals to have postural instability when performing two tasks simultaneously. These findings of decrements in both speech and postural performance reflect a bidirectional dual task interference effect in the participants with PD. The data suggest that one task is not necessarily prioritized over the other, which complements and expands on a previous report which suggested that people with PD may fail to prioritize postural control, and thus put themselves at increased risk of falls (Bloem et al., 2006).

The healthy young and age-matched groups demonstrated no significant change in the speech variables during the dual task condition compared to the single task condition (see table 2). As noted above, it is possible that the speech task was too simple and that it did not require enough attentional resources to affect performance during the dual task condition in these participants. Previous studies of healthy speakers have shown that language generation rather than the simple repetition of an utterance can have a greater impact on limb movements in dual task conditions (Dromey \& Shim, 2008). If the present speech task had required more extensive linguistic processing, a stronger interference effect might have been found, even in 
the healthy individuals. The PD group, however, showed a significant decrease in the formant transition extent and slope for F1 in /כI/ and for F2 in /eI/ when the postural and speaking tasks were performed concurrently. This finding reflects interference in the form of reduced articulatory excursions in the dual task condition. It may be that the cognitive processing resources available to both the younger and older healthy individuals were sufficient to accommodate the performance of both tasks without reduced speech performance, whereas the individuals with PD were challenged to the point of performance decrements because of their neurological deficits.

The postural measures revealed several significant changes for the dual task condition (see table 3). While reaction time increased for all groups, the changes were only significant for the healthy young and participants with PD. This variable was selected as an index of motor planning, and the results thus suggest that more attentional resources were needed to prepare for the postural task when the participants were speaking (Cutson, 1994). The reaction time increase is consistent with previous research which has reported that the addition of a secondary task decreases postural performance in healthy young individuals, healthy older individuals and people with PD (Marchese et al., 2003; Morris et al., 2000).

The heel height variable revealed a greater dual task impact for the two older groups than for the young healthy controls. This compounded the already reduced performance of the older individuals in the single task condition. This finding raises the concern that people with PD or neurologically healthy elderly individuals, who may already be impaired in their postural motor performance, will be disproportionately affected by the addition of a common daily task such as speaking. Heel height variability, on the other hand, was more affected by dual tasking 
in the healthy young participants. An explanation for this finding may lie in an examination of the heel height measure in each group. In the rise to toes task, the higher the heel is off the floor, the more difficult it is to maintain stability, and thus the healthy young controls would naturally have been more susceptible to heel height variability because of the greater elevation above the floor that they achieved. The participants were asked to stay as "high and stable" as they could, and the healthy young group was able to stay much higher despite the increased variability, whereas the older groups sacrificed both heel height and stability. Thus, it can be argued that for all three areas of postural control analyzed in the present study (motor planning, postural coordination, and postural stability) the older groups were affected to a greater degree by the dual task condition than the healthy young group. This supports previous research that has indicated that older individuals with and without PD are impacted to a greater degree than healthy young individuals when confronted with dual task situations (Bloem et al., 2006; Hackney \& Earhart, 2009; Marchese et al., 2003; Plotnik, Giladi, \& Hausdorff, 2009).

Although only a few of the postural variables changed significantly for the dual task condition, table 2 shows that all groups sacrificed some degree of stability. Healthy young individuals do not necessarily need to adopt a posture first strategy until balance is seriously challenged and they are at risk of falling (Barra, Bray, Sahni, Golding, \& Gresty, 2006). In the present study, the dual task condition affected postural performance in the healthy young group, but not to the extent of seriously challenging their stability. Therefore, it could be argued that the healthy young individuals did not have to resort to a posture first strategy. They presumably had enough attentional resources to perform the speech task with only minimal 
stability compromise, and they did not face a priority decision regarding their safety. The same argument could be made for the age-matched group. Even with the addition of a secondary task, postural performance of both control groups was generally superior to the single task measures from the PD group.

On the other hand, the PD group had more difficulty with the dual task condition and likely had their stability significantly challenged. The PD group already had smaller and slower postural movements during the single task condition. They were susceptible to the addition of a second task, thus pushing them closer to losing postural stability. The interference displayed in the diphthong measures in the dual task condition suggests that the dual task processing demands may have exceeded the available attentional resources for this group but not for the older or younger controls. The decrements seen in both speech and postural performance in the PD group suggest that they did not prioritize one task over the other by choosing the less safe posture second strategy (Bloem et al., 2006), since speech was also compromised. Our results suggest that instead of selecting a posture second approach, the group with PD may have failed to select a strategy altogether. This potentially risky lack of priority could contribute to an increased likelihood of falling.

\section{Limitations and Directions for Future Research}

Recognizing a number of limitations in the present study may provide valuable direction in planning future research into dual task performance in PD. The lack of many significant speech variable differences between the speakers with PD and the controls groups may be attributable in part to the modest samples sizes, the lack of more severely dysarthric participants, and individual speaker variability in performance. Thus, a larger sample may have 
allowed the detection of the types of speech differences reported in other studies. The sentence repetition task was somewhat contrived, and may not have been representative of the types of communication situations that can lead to perceptible deficits in the speech of individuals with PD.

The rise to toes task employed in the present study may not have been reflective of the typical postural challenges encountered by people with PD. It may be beneficial in a clinical context to evaluate how they perform under more ecologically valid dual task conditions. A number of dual task assessments can be performed that may be beneficial in determining whether a patient with PD is susceptible to overtaxing their attentional resources, and such assessment protocols may have value in predicting the likelihood that an individual will become worse in their speech performance or possibly fall (Beauchet, Annweiler, Dubost, Allali, Kressig et al., 2009; Zijlstra, Ufkes, Skelton, Lundin-Olsson, \& Zijlstra, 2008).

It may also be valuable to provide training in more effective attentional shifts in this population, because this has been shown to decrease dual task interference effects on gait in people with PD (Baker, Rochester, \& Nieuwboer, 2007; Canning, 2005). Future research could examine the effects of practice on the ability to minimize bidirectional interference in both speech and postural stability tasks.

\section{Summary}

Our research indicates that the prioritizing of simultaneous tasks may be an important issue to address in the rehabilitation of hypokinetic dysarthria and postural instability in individuals with mild to moderate PD. While the concurrent performance of speech and postural stability tasks may be trivial in neurologically healthy persons, this appears not to be 
Dual task interference in PD

the case in PD. 


\section{References}

Ackermann, H. \& Ziegler, W. (1991). Articulatory deficits in parkinsonian dysarthria: an acoustic analysis. Journal of Neurology, Neurosurgery \& Psychiatry, 54, 1093-1098.

Adams, S. G. (1996). Hypokinetic dysarthria in Parkinson's disease. In M.R.McNeil (Ed.), Clinical management of sensorimotor speech disorders (pp. 259-282). New York: Thieme.

Adams, S. G., Weismer, G., \& Kent, R. D. (1993). Speaking rate and speech movement velocity profiles. Journal of Speech and Hearing Research, 36, 41-54.

Baker, K., Rochester, L., \& Nieuwboer, A. (2007). The immediate effect of attentional, auditory, and a combined cue strategy on gait during single and dual tasks in Parkinson's disease. Archives of Physical Medicine and Rehabilitation, 88, 1593-1600.

Barra, J., Bray, A., Sahni, V., Golding, J. F., \& Gresty, M. A. (2006). Increasing cognitive load with increasing balance challenge: Recipe for catastrophe. Experimental Brain Research, 174, 734-745.

Beauchet, O., Annweiler, C., Dubost, V., Allali, G., Kressig, R. W., Bridenbaugh, S. et al. (2009). Stops walking when talking: a predictor of falls in older adults? European Journal of Neurology, 16, 786-795.

Bloem, B. R., Grimbergen, Y. A., van Dijk, J. G., \& Munneke, M. (2006). The "posture second" strategy: A review of wrong priorities in Parkinson's disease. Journal of the Neurological Sciences, 248, 196-204. 
Brown, R. G. \& Marsden, C. D. (1991). Dual task performance and processing resources in normal subjects and patients with Parkinson's disease. Brain, 114 (Pt 1A), 215-231.

Camicioli, R., Oken, B. S., Sexton, G., Kaye, J. A., \& Nutt, J. G. (1998). Verbal fluency task affects gait in Parkinson's disease with motor freezing. Journal of Geriatric Psychiatry and Neurology, 11, 181-185.

Canning, C. G. (2005). The effect of directing attention during walking under dual-task conditions in Parkinson's disease. Parkinsonism and Related Disorders, 11, 95-99.

Cohen, J. (1992). Statistics: A power primer. Psychology Bulletin, 112, 155-159.

Cutson, T. M. (1994). Assessment of motor planning deficits. Topics in Geriatric Rehabilitation, 10, 56-69.

Dromey, C. (2003). Spectral measures and perceptual ratings of hypokinetic dysarthria. Journal of Medical Speech-Language Pathology, 11, 85-94.

Dromey, C., Ramig, L. O., \& Johnson, A. B. (1995). Phonatory and articulatory changes associated with increased vocal intensity in Parkinson disease: a case study. Journal of Speech and Hearing Research, 38, 751-764.

Dromey, C. \& Shim, E. (2008). The effects of divided attention on speech motor, verbal fluency, and manual task performance. Journal of Speech, Language and Hearing Research, 51, 1171-1182. 
Galletly, R. \& Brauer, S. G. (2005). Does the type of concurrent task affect preferred and cued gait in people with Parkinson's disease? Australian Journal of Physiotherapy, 51, 175180.

Gentil, M. \& Pollak, P. (1995). Some aspects of Parkinsonian dysarthria. Journal of Medical Speech-Language Pathology, 3, 221-237.

Hackney, M. E. \& Earhart, G. M. (2009). The effects of a secondary task on forward and backward walking in Parkinson's disease. Neurorehabilitation and Neural Repair.

Ho, A. K., Bradshaw, J. L., \& lansek, R. (2008). For better or worse: The effect of levodopa on speech in Parkinson's disease. Movement Disorders, 23, 574-580.

Holmes, J. D., Jenkins, M. E., Johnson, A. M., Adams, S. G., \& Spaulding, S. J. (2010). Dual-task interference: The effects of verbal cognitive tasks on upright postural stability in Parkinson's disease. Parkinson's Disease, 2010, 1-5.

Lipsey, M. W. (1990). Design sensitivity: Statistical power for experimental research. Newbury Park, CA: Sage Publications.

Marchese, R., Bove, M., \& Abbruzzese, G. (2003). Effect of cognitive and motor tasks on postural stability in Parkinson's disease: a posturographic study. Movement Disorders, $18,652-658$. 
Martin, M., Shinberg, M., Kuchibhatla, M., Ray, L., Carollo, J. J., \& Schenkman, M. L. (2002). Gait initiation in community-dwelling adults with Parkinson disease: comparison with older and younger adults without the disease. Physical Therapy, 82, 566-577.

Morris, M., lansek, R., Smithson, F., \& Huxham, F. (2000). Postural instability in Parkinson's disease: A comparison with and without a concurrent task. Gait and Posture, 12, 205216.

Nakano, K. K., Zubick, H., \& Tyler, H. R. (1973). Speech defects of parkinsonian patients. Effects of levodopa therapy on speech intelligibility. Neurology, 23, 865-870.

Plotnik, M., Giladi, N., \& Hausdorff, J. M. (2009). Bilateral coordination of gait and Parkinson's disease: The effects of dual tasking. Journal of Neurology, Neurosurgery and Psychiatry, $80,347-350$.

Ramig, L. O., Fox, C., \& Sapir, S. (2004). Parkinson's disease: speech and voice disorders and their treatment with the Lee Silverman Voice Treatment. Seminars in Speech and Language, 25, 169-180.

Rochester, L., Nieuwboer, A., Baker, K., Hetherington, V., Willems, A. M., Kwakkel, G. et al. (2008). Walking speed during single and dual tasks in Parkinson's disease: Which characteristics are important? Movement Disorders, 23, 2312-2318.

Weiss, P., Stelmach, G. E., \& Hefter, H. (1997). Programming of a movement sequence in Parkinson's disease. Brain, 120 (Pt 1), 91-102. 
Wu, T. \& Hallett, M. (2008). Neural correlates of dual task performance in patients with Parkinson's disease. Journal of Neurolology, Neurosurgery and Psychiatry, 79, 760-766.

Yogev, G., Giladi, N., Peretz, C., Springer, S., Simon, E. S., \& Hausdorff, J. M. (2005). Dual tasking, gait rhythmicity, and Parkinson's disease: Which aspects of gait are attention demanding? European Journal of Neuroscience, 22, 1248-1256.

Zijlstra, A., Ufkes, T., Skelton, D. A., Lundin-Olsson, L., \& Zijlstra, W. (2008). Do dual tasks have an added value over single tasks for balance assessment in fall prevention programs? A mini-review. Gerontology, 54, 40-49. 
Table 1. Participant demographics

\begin{tabular}{|l|c|c|c|c|}
\hline \multicolumn{1}{|c|}{ Demographic Variable } & \multicolumn{2}{c|}{ Groups } \\
\hline & Healthy Young & Age-Matched & $\begin{array}{c}\text { Parkinson's } \\
\text { Disease }\end{array}$ \\
\hline $\begin{array}{l}\text { Age } \\
\text { Mean }\end{array}$ & 25.50 & & 70.50 & \\
(sd) & $(2.40)$ & $(11.90)$ & 68.70 \\
\hline $\begin{array}{l}\text { Duration of PD } \\
\text { Mean }\end{array}$ & & & $(9.20)$ \\
(sd) & & & 4.11 \\
\hline $\begin{array}{l}\text { Modified Hoehn and Yahr Staging } \\
\text { Median } \\
\text { (min-max) }\end{array}$ & & & $(2.31)$ \\
\hline $\begin{array}{l}\text { Taking Dopamine Replacement Medications } \\
\text { Yes }\end{array}$ & & & 2 \\
No & & & $(1.5-3)$ \\
\hline $\begin{array}{l}\text { Dysarthria Severity (1-10) } \\
\text { Mean } \\
\text { (Min- Max) }\end{array}$ & & & 8 \\
\hline Fall History \\
$\begin{array}{l}\text { Greater than 2 falls in past year } \\
\text { 0-2 falls in past year }\end{array}$ & & & 1 \\
\hline
\end{tabular}


Table 2. Means (and standard deviations) for the acoustic measures from the two diphthongs for each group in the single and dual task conditions with repeated measures ANOVA p-values and effect sizes.

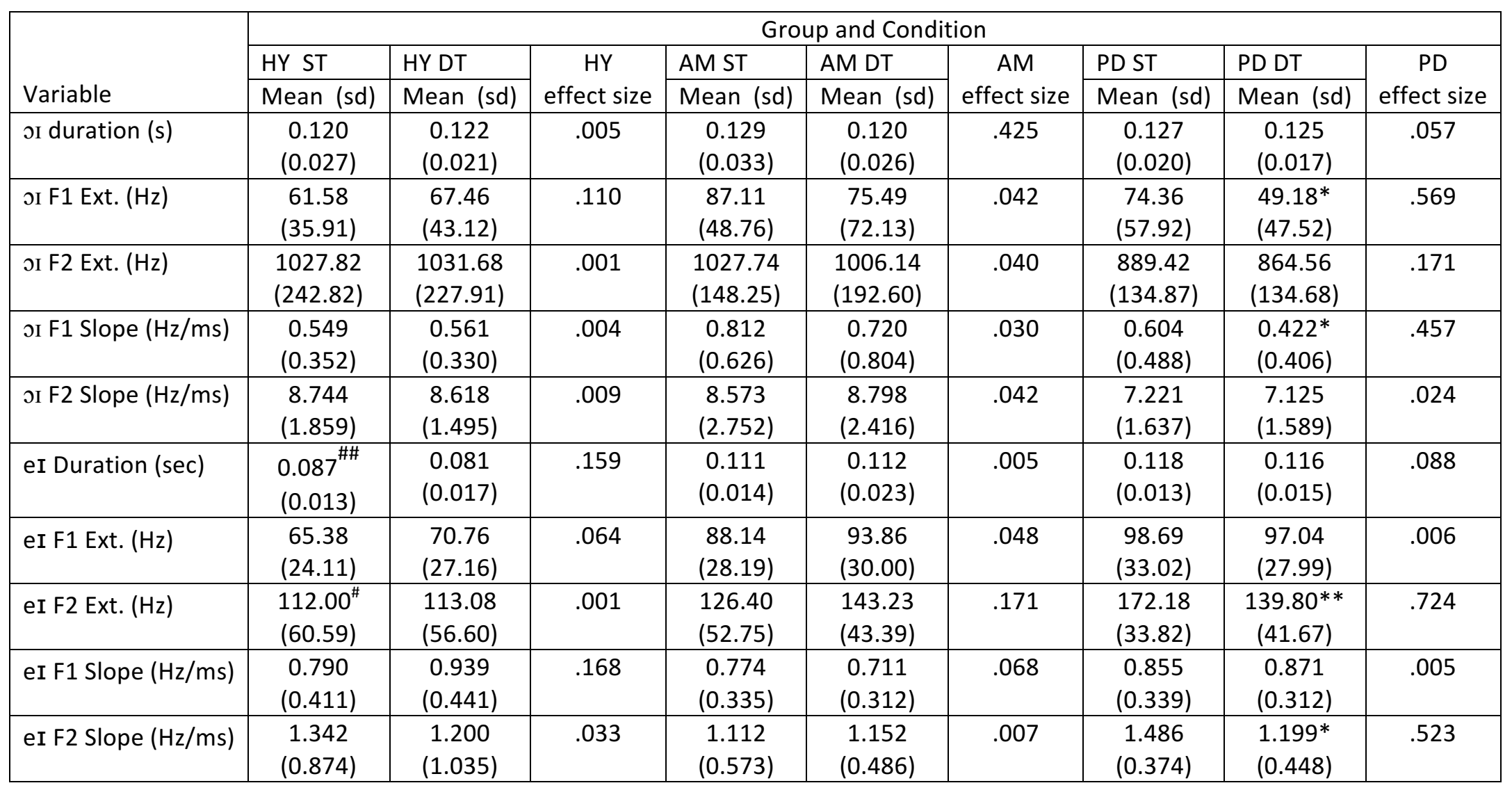

$\mathrm{HY}=$ healthy young controls; $\mathrm{AM}=$ age-matched controls; $\mathrm{PD}=$ Parkinson's disease

Between group performance differences during single task condition: ${ }^{\# \#}=$ HY shorter than PD and AM $(p<.003),{ }^{\#}=H Y$ smaller than $\operatorname{PD}(p=.048)$

Between condition performance differences: ${ }^{*} p<.05 * * p<.01$ 
Table 3. Means (and standard deviations) for the postural measures for each group in the single and dual task conditions with repeated measures ANOVA p-values and effect sizes.

\begin{tabular}{|c|c|c|c|c|c|c|c|c|c|}
\hline \multirow[b]{3}{*}{ Variable } & \multicolumn{9}{|c|}{ Group and Condition } \\
\hline & HY ST & HY DT & HY & AM ST & AM DT & $\mathrm{AM}$ & PD ST & PD DT & $P D$ \\
\hline & Mean (sd) & Mean (sd) & effect size & Mean (sd) & Mean (sd) & effect size & Mean (sd) & Mean (sd) & effect size \\
\hline $\begin{array}{l}\text { RT } \\
\text { (sec) }\end{array}$ & $\begin{array}{c}0.579 \\
(0.127) \\
\end{array}$ & $\begin{array}{l}0.654^{*} \\
(0.115) \\
\end{array}$ & .574 & $\begin{array}{c}0.734 \\
(0.181) \\
\end{array}$ & $\begin{array}{c}0.737 \\
(0.157) \\
\end{array}$ & .002 & $\begin{array}{c}0.697 \\
(0.121) \\
\end{array}$ & $\begin{array}{l}0.791^{*} \\
(0.160) \\
\end{array}$ & .433 \\
\hline $\begin{array}{l}\text { COP Vel } \\
(\mathrm{mm} / \mathrm{sec})\end{array}$ & $\begin{array}{c}439.38^{\#} \\
(90.09)\end{array}$ & $\begin{array}{c}375.07 \\
(100.79)\end{array}$ & .176 & $\begin{array}{c}239.79 \\
(123.30) \\
\end{array}$ & $\begin{array}{c}255.30 \\
(120.35) \\
\end{array}$ & .027 & $\begin{array}{c}201 \\
(140.68)\end{array}$ & $\begin{array}{c}186.06 \\
(138.48)\end{array}$ & .199 \\
\hline COM-COP $(\mathrm{mm})$ & $\begin{array}{l}60.68^{\#} \\
(12.27)\end{array}$ & $\begin{array}{c}55.85 \\
(11.07)\end{array}$ & .081 & $\begin{array}{c}42.05 \\
(16.19)\end{array}$ & $\begin{array}{c}46.68 \\
(14.26)\end{array}$ & .204 & $\begin{array}{c}31.94 \\
(16.07)\end{array}$ & $\begin{array}{c}29.89 \\
(15.55)\end{array}$ & .266 \\
\hline $\begin{array}{l}\mathrm{HH} \\
(\mathrm{mm})\end{array}$ & $\begin{array}{l}96.44^{\#} \\
(17.73) \\
\end{array}$ & $\begin{array}{c}93.08 \\
(17.83) \\
\end{array}$ & .246 & $\begin{array}{c}66.63 \\
(23.97) \\
\end{array}$ & $\begin{array}{c}59.01 * * \\
(25.89) \\
\end{array}$ & .853 & $\begin{array}{c}55.73 \\
(27.08) \\
\end{array}$ & $\begin{array}{l}46.95^{*} \\
(28.13) \\
\end{array}$ & .507 \\
\hline $\mathrm{HH} \mathrm{CV}$ & $\begin{array}{c}0.053 \\
(0.042) \\
\end{array}$ & $\begin{array}{c}0.177^{* *} \\
(0.094) \\
\end{array}$ & .656 & $\begin{array}{c}0.108 \\
(0.162) \\
\end{array}$ & $\begin{array}{c}0.172 \\
(0.157) \\
\end{array}$ & .438 & $\begin{array}{c}0.137 \\
(0.122) \\
\end{array}$ & $\begin{array}{c}0.223 \\
(0.222) \\
\end{array}$ & .151 \\
\hline
\end{tabular}

$\mathrm{HY}=$ healthy young controls; $\mathrm{AM}=$ age-matched controls; $\mathrm{PD}=$ Parkinson's disease

Between group performance differences during single task condition: ${ }^{\#}=$ HY greater than AM and PD $(p<.04)$

Between condition performance differences: ${ }^{*} p<.05 * * p<.01$ 
Dual task interference in PD

\section{Figure Captions}

Figure 1a. Postural data from a single rise to toes task for a healthy young control participant in the single task condition, showing the key dependent variables of heel position (vertical displacement), center of mass position, and center of pressure position as a function of time. For the latter two variables, positive $y$-axis values reflect anterior movement and negative values reflect posterior movement.

Figure 1b. Postural data from a single rise to toes task for a participant with PD in the single task condition. Note reduced COM-COP difference and reduced vertical heel height displacement relative to the healthy young participant illustrated in figure $1 \mathrm{a}$.

Figure 1c. Postural data from a single rise to toes task for a participant with PD in the dual task condition. 
Dual task interference in PD

Figure 1a

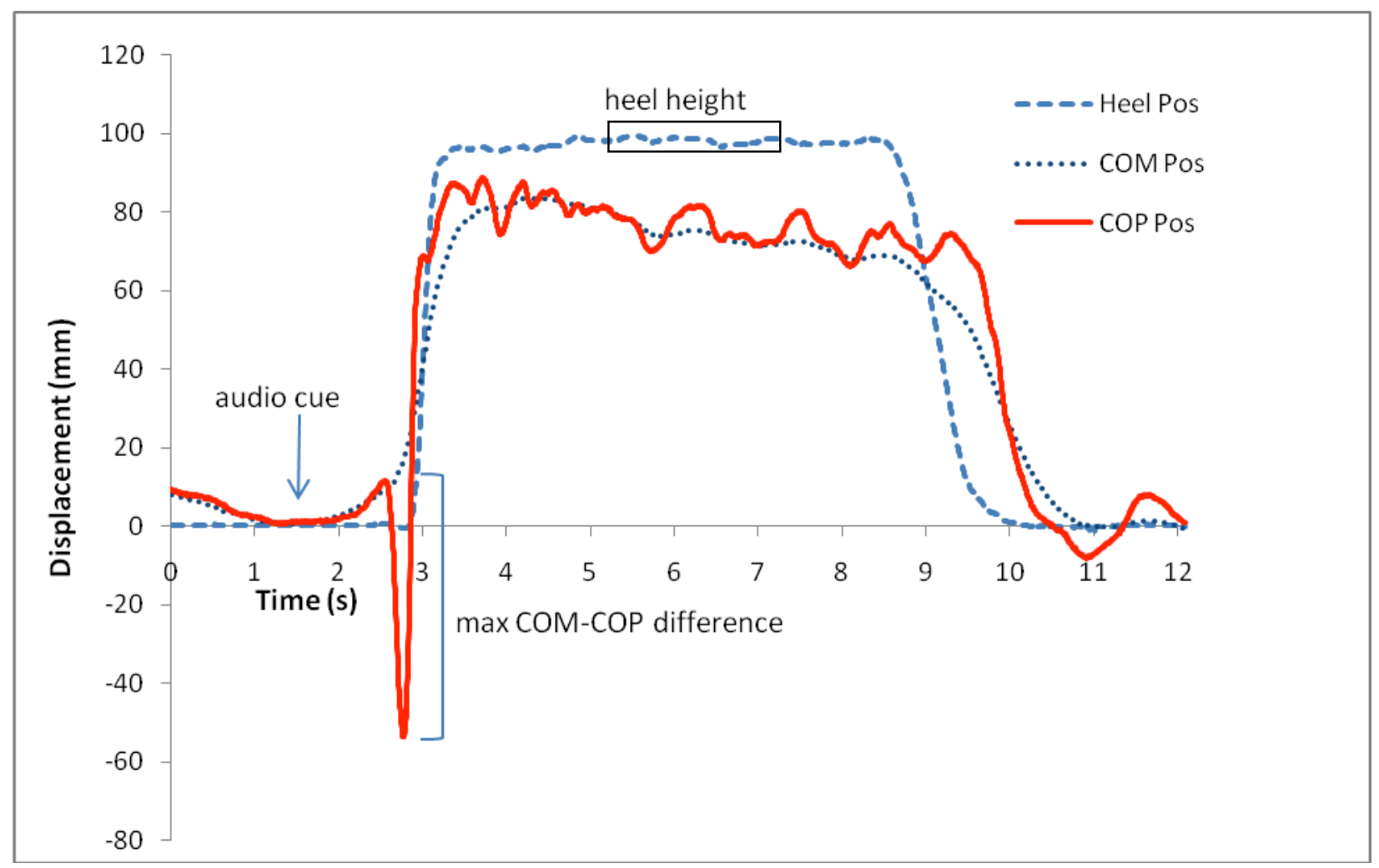


Dual task interference in PD

Figure $1 b$

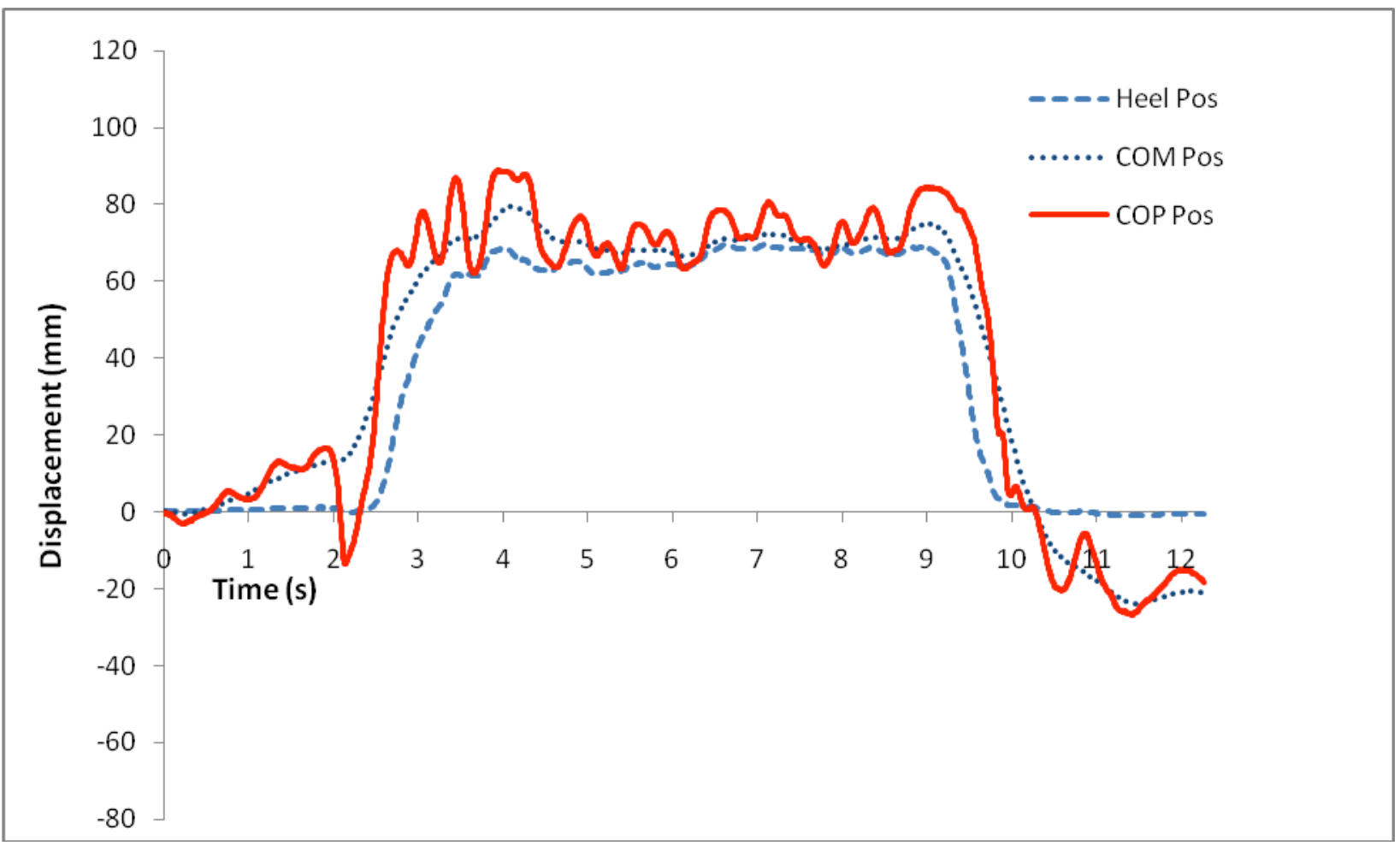


Dual task interference in PD

Figure 1c

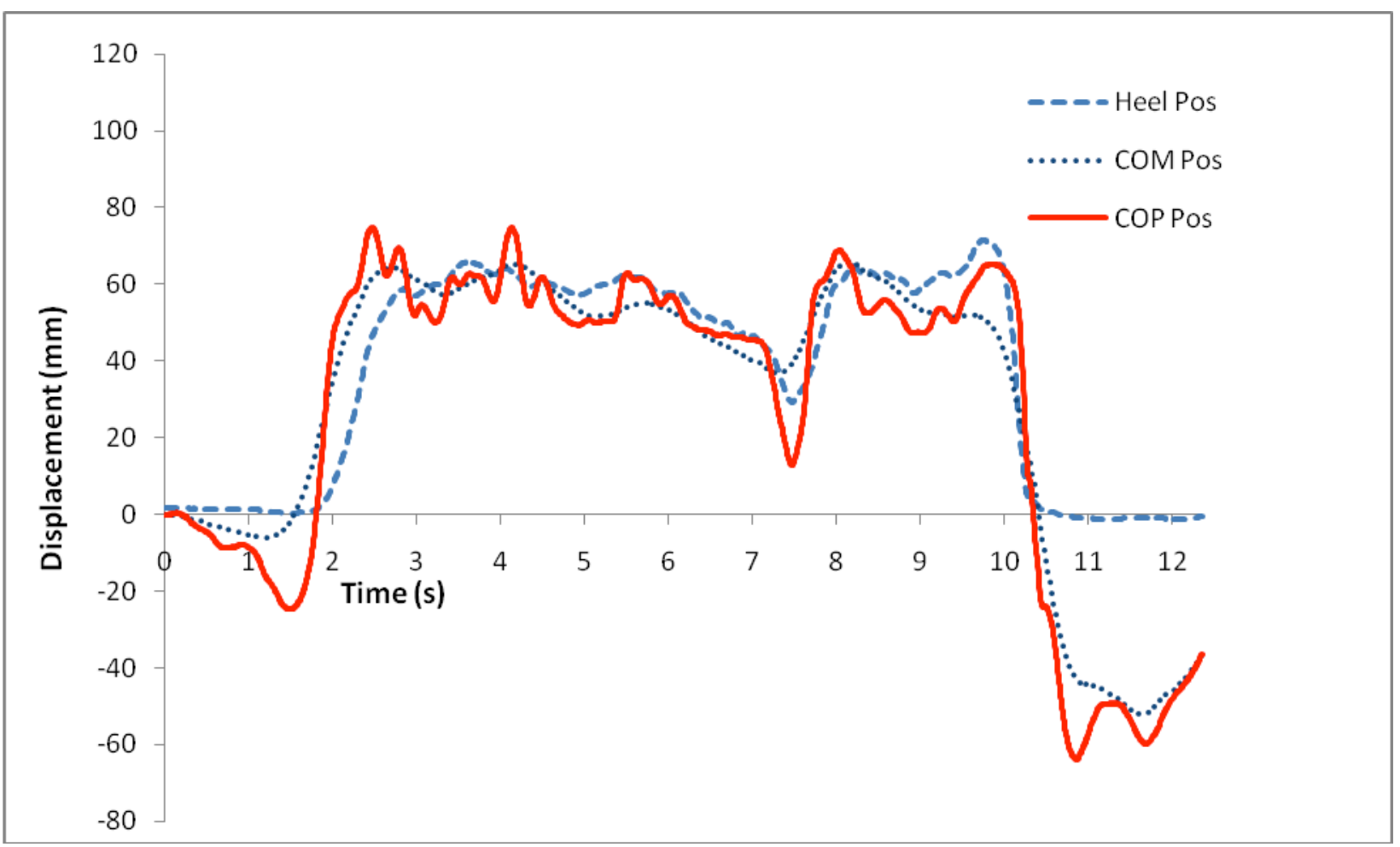

